\title{
Raised Plasma Levels of Asymmetric Dimethylarginine Are Associated with Pathological Type and Predict the Therapeutic Effect in Lupus Nephritis Patients Treated with Cyclophosphamide
}

\author{
Li Zhang Kaichong Zhang Wei Dong Ruizhao Li Renwei Huang \\ Hong Zhang Wanxin Shi Shuangxin Liu Zhuo Li Yuanhan Chen \\ Zhiming Ye Xinling Liang Xueqing Yu
}

Department of Nephrology, Guangdong Provincial People's Hospital, Guangdong Academy of Medical Sciences, Guangzhou, China

\section{Keywords}

Lupus nephritis · Asymmetric dimethylarginine · Biomarker · Cyclophosphamide

\begin{abstract}
Background: Lupus nephritis (LN) is one of the most serious complications of systemic lupus erythematosus (SLE). Asymmetric dimethylarginine (ADMA) has been associated with cardiovascular events in SLE patients and is a strong predictor of the progression of chronic kidney disease. However, whether ADMA can provide a predictive value for the diagnosis and treatment of $L N$ patients remains unclear. This study evaluated the clinical significance of ADMA in LN patients. Methods: Blood samples of 114 patients with LN, 52 patients with primary glomerular disease, and 20 healthy people were collected. Plasma ADMA was measured via enzyme-linked immunosorbent assay. The relationship between plasma ADMA levels and pathological types and renal function and efficacy in LN patients were further analyzed. Results: There was no significant difference in plasma ADMA levels between $L N$ and primary glomerular disease, but both
\end{abstract}

\begin{tabular}{ll}
\hline karger@karger.com & ( ) 2020 The Author(s) \\
Published by S. Karger AG, Basel & Karger \\
Targen access & This article is licensed under the Creative Commons Attribution- \\
NonCommercial-NoDerivatives 4.0 International License (CC BY- \\
NC-ND) (http://www.karger.com/Services/OpenAccessLicense). \\
Usage and distribution for commercial purposes as well as any dis- \\
tribution of modified material requires written permission.
\end{tabular}

were significantly higher than the values in healthy people $(p<0.05)$. Plasma ADMA levels in LN patients were negatively correlated with baseline estimated glomerular filtration rate (eGFR) and serum superoxide dismutase and positively correlated with serum cystatin $C$ and serum $\beta_{2^{-}}$ microglobulin $(p<0.05)$. The plasma ADMA levels of diffuse proliferative $L N$ patients were significantly higher than those of other histopathological classes of LN. High plasma ADMA levels in $\mathrm{LN}$ patients $(\mathrm{OR}=1.012 ; 95 \% \mathrm{Cl} 1.003-1.022 ; p=$ 0.010 ) is a risk factor for diffuse proliferative LN. The area under the receiver operating characteristic $(\mathrm{ROC})$ curve of diagnosing diffuse proliferative LN by plasma ADMA was 0.707 (95\% $\mathrm{Cl} 0.610-0.805)$. The area under the ROC curve of combination with plasma ADMA, serum complement $\mathrm{C} 3$, and eGFR for diffuse proliferative LN was 0.796 (95\% Cl $0.713-$ 0.879), which was significantly higher than that of ADMA, complement $C 3$, and eGFR for diffuse proliferative $L N$ alone, respectively $(p<0.05)$. Low plasma ADMA is an independent protective factor for proliferative $L N$ patients achieving complete remission with cyclophosphamide as induction therapy $(\mathrm{OR}=0.978 ; 95 \% \mathrm{Cl} 0.961-0.996 ; p<0.05)$. Conclusion: High plasma ADMA levels in combination with eGFR and 
complement C3 may be useful to diagnose diffuse proliferative LN. Low plasma ADMA may help to predict complete remission in proliferative $L N$ patients treated with cyclophosphamide as induction therapy. Plasma ADMA may be a new biomarker to determine the pathological type of $L N$ and predict the therapeutic effect. @ 2020 The Author(s)

Published by S. Karger AG, Basel

\section{Introduction}

Systemic lupus erythematosus (SLE) is a chronic complex autoimmune disease that can injure any organ; about 50-60\% SLE patients have a kidney injury, i.e., lupus nephritis (LN), which is a major risk factor for overall mortality in SLE [1-4]. A large number of LN patients will progress to chronic kidney disease (CKD) and about $10 \%$ patients will develop end-stage kidney disease (ESRD) despite the improvements immunosuppressive therapy [5, 6]. One of the strategies to improve the outcome of LN and reduce treatment-related toxicity is to serially evaluate renal disease activity following that initial therapy, which would allow early optimization of immunosuppression [7-9]. Unfortunately, current laboratory parameters, including anti-dsDNA, C3, proteinuria, and estimated glomerular filtration rate (eGFR), are insufficient to predict the histological classification of LN and monitor the treatment response. Although renal biopsy is still the gold standard for histological assessment of LN, its invasiveness as a procedure with potential complications makes it unsuitable for routine monitoring of disease activity and treatment responses [10]. Thus, identification of novel biomarkers that have renal histological lesions and therapeutic guidance or prognostic significance is much needed.

Asymmetric dimethylarginine (ADMA) is a molecule that can inhibit the production of nitric oxide (NO) by blocking the activity of nitric oxide synthetase (NOS). It is regarded as a biomarker of endothelial dysfunction and is elevated in many human disease, such as SLE, CKD, and ESRD [11-13]. In SLE patients, raised plasma ADMA levels are significantly associated with cardiovascular events and organ damage $[13,14]$. Elevated plasma ADMA is an independent predictor of overall mortality in hemodialysis patients and a risk factor for cardiovascular outcomes in ESRD patients [15]. Recently, Fujimi-Hayashida [16] showed that, in patients with IgA nephropathy, ADMA was correlated with the severity of renal tissue damage and the annual reduction rates of eGFR over a 4-year followup time, which could be a predictor of CKD progression. However, whether ADMA can provide a predictive value for the diagnosis and treatment of $\mathrm{LN}$ patients remains unclear. In this study, we aimed to assess the value of ADMA in predicting the pathological type of $\mathrm{LN}$ and the therapeutic response to induction therapy.

\section{Materials and Methods}

\section{Patients and Sample Collection}

A total of 114 patients diagnosed with LN by kidney biopsy were recruited for this investigation from March 2014 to April 2018. All LN patients met the American College of Rheumatology (ACR) revised criteria for SLE. LN plasma samples were collected at the time of renal biopsy. Patients who received any renal replacement therapy or did not have comprehensive basic clinical data at the time of sampling were excluded from the cohort. Blood samples were also obtained in the same manner from patients as follows: minimal change nephrotic syndrome (MCNS), $n=26$; membranous nephropathy (MN), $n=22$; and focal and segmental glomerulosclerosis (FSGS), $n=4$, as disease controls and 20 healthy volunteers without a history of kidney, tumor, cardiovascular, or autoimmune disease as normal controls. All blood samples were centrifuged for $10 \mathrm{~min}$ at 3,000 rpm, and the separated plasma was stored at $-80^{\circ} \mathrm{C}$ until use.

Clinical and laboratory data were collected retrospectively from medical records at the time of renal biopsy. The eGFR was calculated using the CKD-EPI formula [17].

\section{Histopathological Analysis of Kidney Samples}

Histological classification of LN on kidney specimens was performed according to International Society of Nephrology/Renal Pathology Society criteria [18]. Patients presenting class III/III+V were categorized into the focal proliferative LN group. Patients with class IV/IV $+\mathrm{V}$ were categorized into the diffuse proliferative LN group. Patients presenting class $\mathrm{V}$ were categorized into the membranous LN group.

\section{Renal Response Evaluation}

The renal response to induction therapy included complete remission and noncomplete remission. A complete response was defined as a normal or $\leq 25 \%$ decline of the eGFR from baseline and a proteinuria level $<0.5 \mathrm{~g} /$ day. The participants who did not meet the above criteria were reported as having a noncomplete remission.

\section{Enzyme-Linked Immunosorbent Assay}

The ADMA levels of all of the participants were measured using human ADMA enzyme-linked immunosorbent assay kits according to the manufacturer's instructions (csb-e09298h; CUSABIO, Wuhan, China).

\section{Statistics Analyses}

The Kolmogorov-Smirnov test was used to confirm whether the data conformed to a normal distribution. If data were normally distributed, quantitative data were expressed as means \pm SD. The Student $t$ test was used for comparisons between 2 groups. Data that had a nonnormal distribution were expressed as medians $\left(P_{25}\right.$, $\left.P_{75}\right)$, and Mann-Whitney $U$ test was used for comparisons between the 2 groups; the Kruskal-Wallis test was used for comparisons 
Table 1. Demographic and clinical data of the study population

\begin{tabular}{|c|c|c|c|c|}
\hline & LN & $\begin{array}{l}\text { Primary glomerular } \\
\text { disease }\end{array}$ & $\begin{array}{l}\text { Healthy } \\
\text { people }\end{array}$ & $p$ value \\
\hline Age, years & $34(26,46)$ & $35(24,52)$ & $27(25,35)$ & 0.132 \\
\hline Females, $n(\%)$ & $97(85.1)^{\mathrm{b}}$ & $30(57.7)^{\mathrm{a}, \mathrm{b}}$ & $15(75.0)$ & 0.001 \\
\hline $\mathrm{eGFR}, \mathrm{mL} /\left(\mathrm{min} / 1.73 \mathrm{~m}^{2}\right)$ & $87(45,117)$ & $106(92,123)$ & & 0.001 \\
\hline Serum creatinine, $\mu \mathrm{mol} / \mathrm{L}$ & $78(57,139)$ & $66(56,84)$ & & 0.014 \\
\hline Serum $\beta_{2}$-microglobulin, mg/L & $4.61(2.78,7.33)$ & $1.84(1.51,2.16)$ & & 0.000 \\
\hline $\mathrm{SOD}, \mathrm{U} / \mathrm{mL}$ & $90 \pm 31$ & - & & - \\
\hline Serum cystatin $\mathrm{C}, \mathrm{mg} / \mathrm{L}$ & $1.34(1.00,2.21)$ & $0.84(0.73,0.99)$ & & 0.000 \\
\hline SLEDAI & $16(12,20)$ & - & & - \\
\hline Complement C3, mg/L & $525 \pm 236$ & - & & - \\
\hline Cholesterol, $\mathrm{mmol} / \mathrm{L}$ & $6.0(4.8,7.9)$ & $9.2(6.6,11.7)$ & & 0.000 \\
\hline Serum albumin, g/L & $24(18,29)$ & $23(17,32)$ & & 0.972 \\
\hline 24-h urine albumin, mg & $2,076(932,3,199)$ & $1,555(501,3,694)$ & & 0.460 \\
\hline 24 -h urine protein, $\mathrm{mg}$ & $3,379(1,737,6,321)$ & $3,930(1,223,9565)$ & & 0.200 \\
\hline
\end{tabular}

Data are presented as means \pm SD or medians $\left(P_{25}, P_{75}\right)$ unless otherwise stated. ${ }^{a}$ Compared with LN group, $p<0.05$. ${ }^{\mathrm{b}}$ Compared with the healthy group, $p>0.05$. SOD, superoxide dismutase.

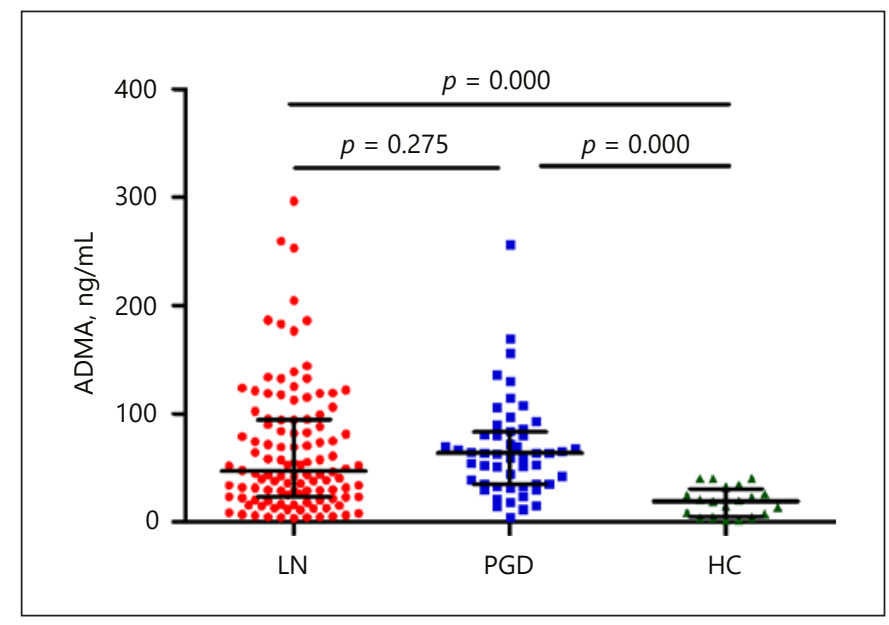

Fig. 1. Comparison of plasma ADMA levels among LN, primary glomerular disease. and healthy people. Plasma ADMA levels in the $\mathrm{LN}$ group $(46.87 \mathrm{ng} / \mathrm{mL}[23.11,94.41])$ and in the primary glomerular disease group $(63.33 \mathrm{ng} / \mathrm{mL}[34.78,83.28])$ showed no statistical significance, but both of them were higher than that in healthy people $(19.28 \mathrm{ng} / \mathrm{mL}[4.91,30.50])(p<0.05)$. PGD, primary glomerular disease; $\mathrm{HC}$, healthy people.

among groups. Categorical data were expressed as frequencies and percentages, and the Pearson $\chi^{2}$ test was used for comparison between groups. Spearman analysis was used to analyze the correlation between plasma ADMA levels and clinical data. Binary logistic regression analysis was used to further analyze the correlations between plasma ADMA levels and clinical data. The receiver operating characteristic (ROC) curve and area under the curve were used to assess the diagnostic value of each biomarker as well as the cutoff value, sensitivity, and specificity. $p<0.05$ was considered statistically significant. All statistical analyses were performed using SPSS version 20.0 for Windows.

\section{Results}

\section{Patient Characteristics}

Samples from 114 renal biopsy-proven LN patients were included in this study. Similarly, samples were collected from 52 patients with MCD, MN, and FSGS as disease controls, and from 20 healthy volunteers as a normal control. Patient profiles and clinical data are shown in Table 1.

\section{Levels of ADMA in LN Patients and Controls}

The serum levels of ADMA in the different groups are compared in Figure 1. There was no significant difference in plasma ADMA levels between LN patients (46.87 ng/ $\mathrm{mL}[23.11,94.41])$ and primary glomerular disease patients $(63.33 \mathrm{ng} / \mathrm{mL}[34.78,83.28])$, but both groups had significantly higher levels compared to the healthy control group $(19.28 \mathrm{ng} / \mathrm{mL}[4.91,30.50])(p<0.05)$.

LN patients were divided into focal proliferative (ISN/ RPS 2003 class III/III+V), diffuse proliferative (ISN/RPS 2003 class IV/IV+V), and nonproliferative (ISN/RPS 2003 class V) groups based on the renal biopsy reports. A total of 84 proliferative cases were analyzed in this study. The baseline data and plasma ADMA levels of the focal $\mathrm{LN}$, diffuse LN, and membranous LN groups are shown, 
Table 2. Comparison of basic data between proliferative LN and membranous LN

\begin{tabular}{|c|c|c|c|c|}
\hline & $\begin{array}{l}\text { Focal proliferative LN } \\
(n=28)\end{array}$ & $\begin{array}{l}\text { Diffuse proliferative LN } \\
(n=56)\end{array}$ & $\begin{array}{l}\text { Membranous LN } \\
(n=24)\end{array}$ & $p$ value \\
\hline Age, years & $34(24,50)$ & $34(26,44)$ & $41(26,47)$ & 0.698 \\
\hline eGFR & $96(52,122)$ & $60(40,104)$ & $114(92,123)^{\mathrm{a}}$ & 0.001 \\
\hline SLEDAI & $16(12,21)$ & $16(12,20)$ & $13(9,16)^{\mathrm{a}}$ & 0.023 \\
\hline Complement C3, mg/L & $589(390,717)$ & $387(321,585)$ & $703(446,810)^{\mathrm{a}}$ & 0.001 \\
\hline Serum albumin, g/L & $27(20,31)$ & $22(18,28)$ & $23(15,31)$ & 0.139 \\
\hline 24-h urine albumin,mg & $2,076(873,2,871)$ & $2,456(1,231,3,273)$ & $1,784(530,3,733)$ & 0.244 \\
\hline 24-h urine protein, mg & $3,429(1,725,6,764)$ & $3,746(2,236,6,459)$ & $2,547(965,5,362)$ & 0.373 \\
\hline
\end{tabular}

Data are represented as medians $\left(P_{25}, P_{75}\right)$. ${ }^{\text {a }}$ Compared with the diffuse proliferative LN group, $p<0.05$.

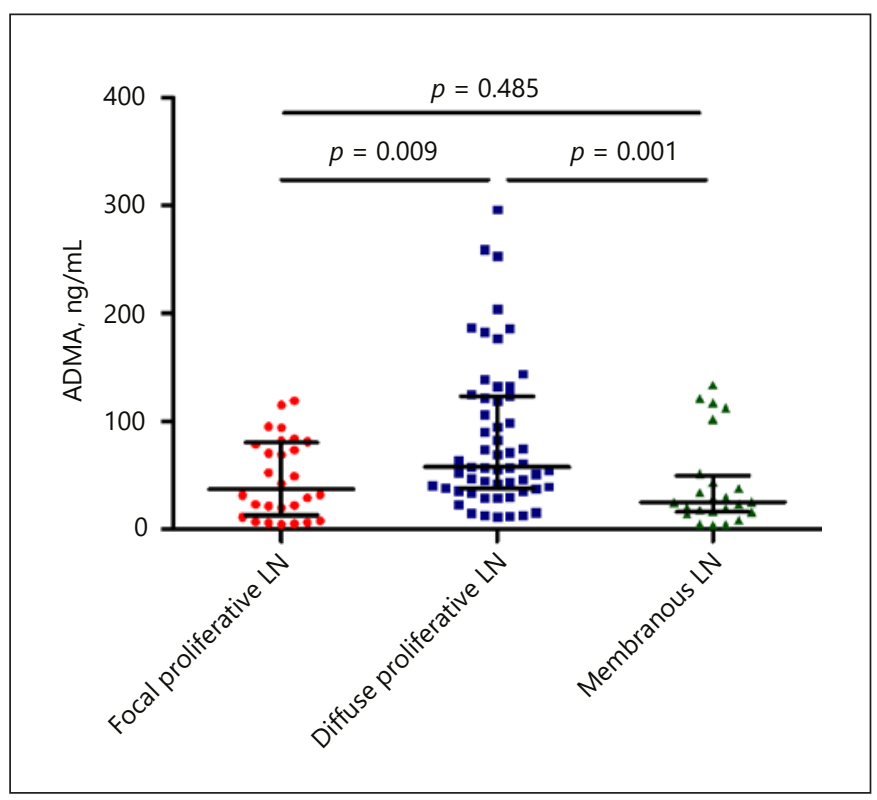

Fig. 2. Comparison of plasma ADMA levels among focal LN, diffuse LN, and membranous LN patients. Plasma ADMA levels of diffuse proliferative LN $(57.80 \mathrm{ng} / \mathrm{mL}[37.91,123.23])$ were significantly separately higher than those of focal proliferative LN $(37.05 \mathrm{ng} / \mathrm{mL}[13.29,80.59])$ and membranous LN $(25.24 \mathrm{ng} / \mathrm{mL}$ $[16.13,49.78])(p<0.01)$.

respectively, in Table 2 and Figure 2. There was no significant difference in age or sex ratio among the 3 groups. The plasma ADMA levels of diffuse proliferative LN patients $[57.80 \mathrm{ng} / \mathrm{mL}(37.91,123.23)]$ were significantly higher than those of focal proliferative $\mathrm{LN}[37.05 \mathrm{ng} / \mathrm{mL}$ $(13.29,80.59)]$ and membranous $\mathrm{LN}[25.24 \mathrm{ng} / \mathrm{mL}(16.13$, $49.78)](p<0.05)$ patients, respectively.
Table 3. Relationship between plasma ADMA and baseline clinical characteristics in $\mathrm{LN}$ patients

\begin{tabular}{lrc}
\hline & $r_{\mathrm{s}}$ & $p$ value \\
\hline Age (years) & 0.211 & 0.024 \\
SLEDAI & 0.160 & 0.088 \\
Serum creatinine $(\mu \mathrm{mol} / \mathrm{L})$ & 0.342 & 0.000 \\
eGFR $\left(\mathrm{mL} /\left[\mathrm{min} / 1.73 \mathrm{~m}^{2}\right]\right)$ & -0.355 & 0.000 \\
Serum $\beta_{2}$-microglobulin $(\mathrm{mg} / \mathrm{L})$ & 0.382 & 0.000 \\
Serum cystatin C $(\mathrm{mg} / \mathrm{L})$ & 0.391 & 0.000 \\
SOD $(\mathrm{U} / \mathrm{mL})$ & -0.359 & 0.011 \\
Complement C3 $(\mathrm{mg} / \mathrm{L})$ & -0.145 & 0.124 \\
Cholesterol $(\mathrm{mmol} / \mathrm{L})$ & -0.049 & 0.607 \\
Serum albumin $(\mathrm{g} / \mathrm{L})$ & 0.024 & 0.796 \\
24-h urine protein $(\mathrm{mg})$ & 0.042 & 0.658 \\
24-h urine albumin $(\mathrm{mg})$ & 0.139 & 0.140 \\
\hline
\end{tabular}

SOD, superoxide dismutase.

\section{Correlation Analysis between Plasma ADMA and Clinical Parameters of LN Patients}

Bivariate correlation analysis was performed to explore the correlations between plasma ADMA levels and clinical parameters in LN patients. As demonstrated in Table 3, plasma ADMA was positively correlated with serum creatinine, serum $\beta_{2}$-microglobulin, and serum cystatin $\mathrm{C}(p<0.001)$ and negatively correlated with eGFR and serum superoxide dismutase $(p<0.05)$. There was no correlation between plasma ADMA and SLE disease activity index (SLEDAI) score, complement C3, cholesterol, serum albumin, or 24 -h urinary protein/albumin in LN patients $(p>0.05)$. 
Table 4. Logistic regression analysis of diffuse $\mathrm{LN}$ in patients with $\mathrm{LN}$

\begin{tabular}{|c|c|c|c|c|c|c|}
\hline & \multicolumn{3}{|c|}{ Univariate logistic regression } & \multicolumn{3}{|c|}{ Multivariate logistic regression } \\
\hline & OR & $95 \% \mathrm{CI}$ & $p$ value & OR & $95 \% \mathrm{CI}$ & $p$ value \\
\hline ADMA & 1.015 & $1.006-1.024$ & 0.001 & 1.012 & $1.003-1.022$ & 0.010 \\
\hline Age & 1.001 & $0.972-1.030$ & 0.973 & - & & \\
\hline Female & 0.949 & $0.337-2.679$ & 0.922 & - & & \\
\hline eGFR & 0.980 & $0.969-0.991$ & 0.000 & 0.984 & $0.973-0.996$ & 0.010 \\
\hline SLEDAI & 1.054 & $0.983-1.131$ & 0.140 & - & & \\
\hline Complement C3 & 0.997 & $0.995-0.999$ & 0.001 & 0.997 & $0.995-0.999$ & 0.004 \\
\hline Cholesterol & 0.990 & $0.836-1.171$ & 0.903 & - & & \\
\hline Serum albumin & 0.960 & $0.907-1.016$ & 0.162 & - & & \\
\hline 24-h urine protein & 1.000 & $1.000-1.000$ & 0.493 & - & & \\
\hline
\end{tabular}

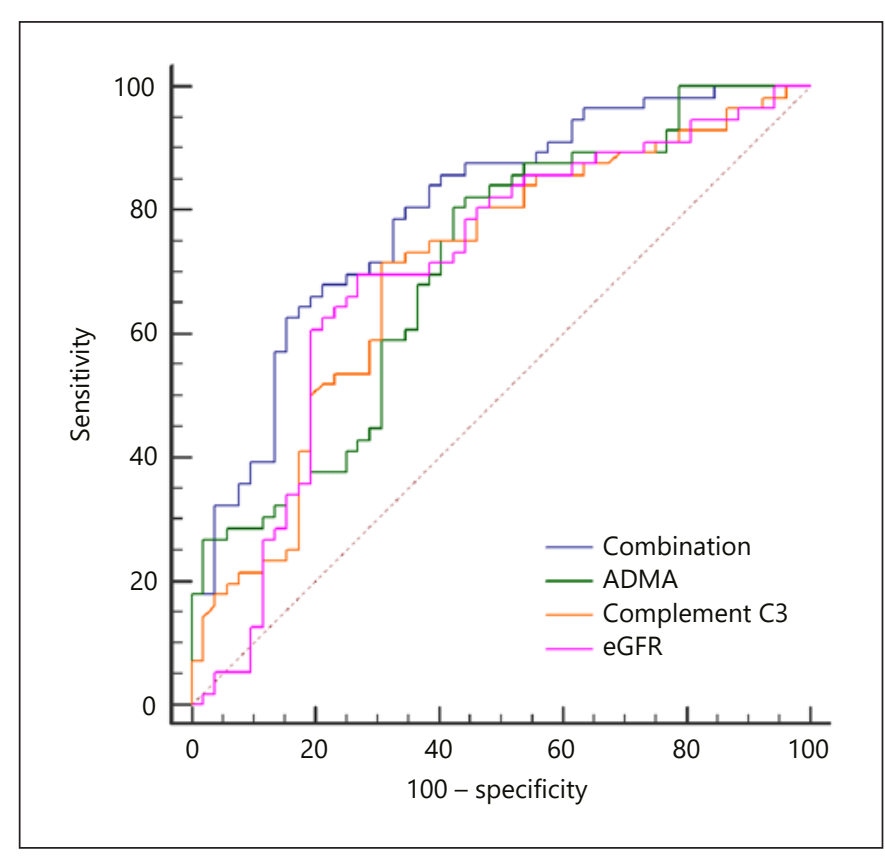

Fig. 3. ROC curve of plasma ADMA, eGFR, complement C3, and their combination for diagnosis of diffuse proliferative LN, compared with the AUC of the combination with plasma ADMA, eGFR, and complement C3 $(p<0.05)$. The AUC of the combination with plasma ADMA, eGFR, and complement $\mathrm{C} 3$ was higher than that of plasma ADMA, eGFR, and complement C3 separately for diagnosing diffuse proliferative LN.

Plasma ADMA levels were significantly higher in diffuse proliferative LN (class IV/IV $+\mathrm{V}$ ) patients. To further analyze the risk factors for diffuse proliferative LN patients, we performed a univariate logistic regression analysis. The results showed that a high plasma ADMA level $(\mathrm{OR}=1.015 ; 95 \%$ CI $1.006-1.024 ; p=0.001)$, a low eGFR level $(\mathrm{OR}=0.980 ; 95 \%$ CI $0.969-0.991 ; p=0.000)$, and a low complement C3 level (OR $=0.997$; $95 \%$ CI $0.995-$ $0.999 ; p=0.001$ ) are risk factors for diffuse proliferative LN. Further multivariate logistic regression analysis demonstrated that plasma ADMA, eGFR, and complement C3 are independent risk factors for the occurrence of diffuse proliferative LN ( $p<0.05$; Table 4$)$.

Next, ROC curves was constructed to assess the diagnostic performance of ADMA, eGFR, and complement $\mathrm{C} 3$ in diffuse proliferative $\mathrm{LN}$. The area under the ROC curve (AUC) of ADMA is 0.707 (95\% CI $0.61-0.81$, cutoff $=33.79 \mathrm{ng} / \mathrm{mg}$, sensitivity $=80.4 \%$, specificity $=$ 57.7\%). eGFR showed an AUC of 0.703 (95\% CI 0.60 $0.81,69.6 \%$ sensitivity, $73.1 \%$ specificity). The AUC value of $0.701(95 \% \mathrm{CI}=0.60$ to 0.80$)$ with $71.4 \%$ sensitivity and $69.2 \%$ specificity was produced by complement $\mathrm{C} 3$ (Fig. 3). Furthermore, the AUC of the combination of plasma ADMA, eGFR, and complement $\mathrm{C} 3$ was, respectively, higher than that of plasma ADMA, eGFR, and complement C3 (Table 5; Fig. 3 ) ( $p<0.05)$. Taken together, these data indicate that plasma AMDA could be a useful LN biomarker for the prediction of diffuse proliferative LN.

Plasma ADMA in Predicting the Renal Response

in Proliferative LN Patients Treated with

Cyclophosphamide as Induction Therapy

A total of 50 patients with (including class III/III+V, IV/IV+V LN) were treated with cyclophosphamide as induction therapy; 27 of them patients had complete remission after 6 months of induction therapy and 23 had noncomplete remission. Baseline plasma ADMA and 24-h urinary albumin in the complete response group were significantly lower than those in the noncomplete re- 
Table 5. The AUC for diagnosing diffuse proliferative LN

\begin{tabular}{lllllll}
\hline Indicator & AUC & $95 \%$ CI & $p$ value & Cut-off & Sensitivity, \% & Specificity, \% \\
\hline ADMA & $0.707^{\mathrm{a}}$ & $0.610-0.805$ & 0.000 & $>33.79$ & 80.4 & 57.7 \\
eGFR & $0.703^{\mathrm{a}}$ & $0.601-0.805$ & 0.000 & $<81.89$ & 69.6 & 73.1 \\
Complement C3 & $0.701^{\mathrm{a}}$ & $0.601-0.801$ & 0.000 & $<497$ & 71.4 & 69.2 \\
Combination & 0.796 & $0.713-0.879$ & 0.000 & & 62.5 & 84.6 \\
\hline
\end{tabular}

${ }^{\text {a }}$ Compared with the AUC of the combination of plasma ADMA, eGFR, and complement C3 $(p<0.05)$.

Table 6. Comparison of basic data of proliferative LN with different therapeutic effects treated with cyclophosphamide

\begin{tabular}{lccc}
\hline & $\begin{array}{l}\text { Complete remission } \\
(n=27)\end{array}$ & $\begin{array}{l}\text { Noncomplete remission } \\
(n=23)\end{array}$ & $p$ value \\
\hline Plasma ADMA, ng/mL & $57.24(28.96,81.22)$ & $83.81(42.38,123.73)$ & 0.033 \\
Age, years & $39(23,51)$ & $41(31,53)$ & 0.227 \\
eGFR, mL/(min/1.73m $\left.{ }^{2}\right)$ & $73(47,111)$ & $51(34,102)$ & 0.076 \\
SLEDAI & $16(13,20)$ & $16(12,20)$ & 0.845 \\
Complement C3, mg/L & $406(311,596)$ & $457(349,607)$ & 0.465 \\
Serum albumin, g/L & $25(20,29)$ & $20(17,26)$ & 0.007 \\
24-h urine albumin, mg & $2,064(807,2,944)$ & $2,826(1,758,4,412)$ & 0.007 \\
24-h urine protein, mg & $3,836(1,281,6,459)$ & $4,367(2,664,8,219)$ & 0.100
\end{tabular}

Data are represented as medians $\left(P_{25}, P_{75}\right)$.

Table 7. Logistic regression analysis of complete remission in patients with proliferative LN treated with cyclophosphamide

\begin{tabular}{|c|c|c|c|c|c|c|}
\hline & \multicolumn{3}{|c|}{ Univariate logistic regression } & \multicolumn{3}{|c|}{ Multivariate logistic regression } \\
\hline & OR & $95 \% \mathrm{CI}$ & $p$ & OR & $95 \% \mathrm{CI}$ & $p$ \\
\hline Plasma ADMA & 0.984 & $0.971-0.998$ & 0.026 & 0.978 & $0.961-0.996$ & 0.015 \\
\hline Serum albumin & 1.147 & $1.026-1.281$ & 0.016 & 1.122 & $0.970-0.996$ & 0.121 \\
\hline 24-h urine albumin & 0.999 & $0.999-1.000$ & 0.006 & 1.000 & $0.999-1.000$ & 0.092 \\
\hline
\end{tabular}

sponse group ( $p<0.05$; Table 6$)$. The baseline serum albumin level in the complete response group was significantly higher than that in the noncomplete response group $(p<0.05)$. There were no significant differences in age, eGFR, SLEDAI score, complement C3, or 24-h urinary protein between the 2 groups ( $p>0.05$; Table 6 ).

A univariate logistic regression analysis of protective factors for proliferative LN (including class III/III+V, IV/ $\mathrm{IV}+\mathrm{V}$ LN) patients treated with cyclophosphamide as induction therapy to achieve complete remission showed that low plasma ADMA (OR $=0.984 ; 95 \%$ CI 0.971-0.998; $p=0.026)$, low 24 -h urinary albumin $(\mathrm{OR}=0.999 ; 95 \% \mathrm{CI}$
$0.999-1.000 ; p=0.006)$, and high serum albumin (OR = $1.147 ; 95 \%$ CI $1.026-1.281 ; p=0.016$ ) were protective factors for complete remission in proliferative LN patients treated with cyclophosphamide (Table 7). The multivariate logistic regression analysis showed that low plasma ADMA (OR $=0.978 ; 95 \%$ CI 0.961-0.996; $p=0.015)$ was an independent protective factor for proliferative LN patients achieving complete response via treatment with cyclophosphamide in the induction therapy period (Table 7). These data suggest that low plasma ADMA can be a useful biomarker for predicting complete remission in patients with proliferative LN treated with cyclophosphamide. 


\section{Discussion}

Prior studies have demonstrated the potential value of plasma ADMA in cardiovascular events and organ damage in SLE, IgA, and hemodialysis patients [13-16]. In this study, we examined the role of ADMA in predicting the pathological type of LN and the renal response to induction therapy with cyclophosphamide. The results indicated that plasma ADMA levels in LN patients were higher than those in healthy individuals. Plasma ADMA levels in combination with eGFR and complement C3 may help to determine diffuse proliferative LN. Low plasma ADMA is an independent protective factor for proliferative LN patients achieving complete remission with cyclophosphamide as induction therapy. Plasma ADMA may be a new biomarker to determine the pathological type of LN and predict the therapeutic effect.

ADMA is an amino acid, it widely exists in cells and tissues, and it decreases the synthesis of NO called endogenous NOS inhibitor [19]. It is involved in the pathological physiological process of vascular endothelial cell dysfunction, which is associated with diseases such as cardiovascular disease and CKD [20-23]. ADMA is a predictor of the progression of CKD [24]. It has been reported that increased plasma ADMA levels in SLE patients are associated with arterial stiffness and the incidence of cardiovascular events $[13,25]$. In this study, we found that plasma ADMA levels in LN patients were significantly higher than those in healthy individuals. We also tested the plasma ADMA levels in patients with primary glomerular disease (including MCD, FSGS, and MN) as a disease control. The results showed that the plasma ADMA levels of patients with primary glomerular disease were also higher than those of the healthy control group, but no obvious difference was found between LN and primary glomerular disease patients, indicating that increased plasma ADMA levels are not specific to patients with LN. This is consistent with previous reports that plasma ADMA levels are increased in IgA nephropathy patients [16].

To evaluate the disease activity of ADMA in LN patients, we further analyzed the correlation between plasma ADMA level and SLEDAI score and complement C3 in LN patients. Spearman correlation analysis showed no correlation between plasma ADMA level and SLEDAI score and complement C3 in LN patients, which is consistent with literature reports in SLE patients [25]. SLEDAI and complement C3 are indicators for evaluating SLE activity, without organ specificity. A high plasma ADMA level may be more specific for specific organ damage in SLE (such as kidney and cardiovascular). Further analysis indicated that plasma ADMA levels in LN patients were correlated with serum creatinine, cystatin C, and $\beta_{2}$-microglobulin levels, which can reflect kidney function. It has been reported that there is no correlation between the serum ADMA value and the 24-h creatinine clearance rate in IgA nephropathy patients [16]. Only about $5 \%$ of ADMA is present in urine after intravenous injection in rabbits [26]. ADMA is mainly metabolized by dimethylarginine dimethylaminohydrolase (DDAH) [27], and only a small amount is excreted in urine. DDAH is widely distributed throughout the body, particularly in the liver and kidney; circulating ADMA can be transported to those organs for enzymatic degradation [28, 29]. Thus, the level of plasma ADMA mainly depends on the expression and activity of DDAH and not on a decreased kidney excretion capacity. Elevated plasma ADMA levels may indicate renal dysfunction in LN patients, but the mechanism is unknown.

Current biomarkers for $\mathrm{LN}$ such as proteinuria, serum creatinine, anti-dsDNA, and complement levels lack sensitivity and specificity to detect renal disease activity in $\mathrm{LN}$. Although proteinuria is the main criteria for response to treatment, it may be from fibrosis/scarring. The amount of proteinuria is not always proportional to the degree of kidney damage. Complements can also decrease in immune-complex-mediated lesions, such as vasculitis, despite no renal disease activity. Therefore, sensitive biomarkers reflecting intrarenal damage are needed. Our findings revealed that the plasma ADMA levels of diffuse proliferative LN patients were significantly higher than those of focal proliferative LN and membranous LN patients. Further analysis showed that a high plasma ADMA level is the risk factor for diffuse proliferative LN and the AUC of plasma ADMA for diagnosing diffuse proliferative $\mathrm{LN}$ is 0.707. Moreover, the combination of ADMA, eGFR, and complement C3 showed increased power to determine diffuse proliferative $\mathrm{LN}$, providing a noninvasive means for pathological diagnosis in LN patients. The mechanism of the significant increase in plasma ADMA level in patients with diffuse proliferative $\mathrm{LN}$ is not clear. ADMA is a marker for endothelial dysfunction, which directly inhibits the synthesis of $\mathrm{NO}$ (one of the most important functions of endothelial cells). NO synthesis inhibition in the kidney may lead to reduced glomerular blood flow, an increase in the vascular resistance of the afferent and efferent arterioles, reduced ultrafiltration and glomerular filtration rates, and production of reactive oxygen species [30].

It has been shown that ADMA may be involved in peritubular capillary loss and tubulointerstitial fibrosis, 
thereby contributing to the progression of CKD [31]. Pathological changes such as inflammation, immune complex formation/deposition, complement activation, cell proliferation, glomerular sclerosis, and tubular interstitial ischemia have been observed in the kidneys of LN patients, especially when the endothelial cell proliferation of diffuse proliferative LN is more severe [32]. $\mathrm{DDAH}$ is present in the proximal tubules, the glomerulus, Bowman's capsule, and the endothelium of blood vessels, and ADMA can be metabolized by DDAH [33]. The renal tissue damage in LN, especially in diffuse proliferative LN, may destroy or reduce the activity of DDAH, subsequently affecting the degradation of ADMA in the kidneys and ultimately leading to increased plasma levels. However, further experiments are required to confirm this.

Cyclophosphamide is still a commonly used induction therapy for class III and IV LN $[8,9]$. Our results suggest that in proliferative LN (including class III/III+V, IV/ IV $+V$ LN) the levels of plasma ADMA in patients in complete remission after treatment with cyclophosphamide as induction therapy were significantly lower than those in those patients with noncomplete remission. A low plasma ADMA level was an independent protective factor for renal complete remission in proliferative LN patients receiving cyclophosphamide as induction therapy. A high plasma ADMA level maybe indicate more severe renal damage and a poor therapeutic effect. In this study, the follow-up time was too short to assess whether patients with high baseline plasma ADMA levels would need more time to achieve renal complete remission. Because of the small number of cases treated with mycophenolate mofetil, we could not analyze whether a high level of plasma ADMA was an independent risk factor for failure of mycophenolate mofetil in the treatment of proliferative LN. The results of this study suggest that proliferative LN patients with high baseline plasma ADMA levels may not benefit from using cyclophosphamide as induction therapy; this provides a noninvasive biomarker to predict the effect of LN patients and help doctors to make a better therapeutic regimen.

The significance of this study is that elevated plasma ADMA levels in LN patients may assist in the diagnosis of diffuse proliferative LN and predict the efficacy of cyclophosphamide as induction therapy for proliferative LN. This study has also certain limitations. It is a retrospective study and so we cannot clarify the mechanism and causal relationship between plasma ADMA level and renal tissue injury or renal function decline in $\mathrm{LN}$ patients. Second, the sample size was small and the follow- up time was short. Third, blood samples of SLE patients without LN were absent, so the difference in plasma ADMA levels between SLE without LN and LN could not be compared. It is necessary to conduct a multicenter, prospective clinical trial with long follow-up time to verify the results of this study and its clinical applicability.

In summary, this is the first study to demonstrate that plasma ADMA levels in LN patients are significantly higher than in healthy individuals. Plasma ADMA levels in patients with diffuse proliferative LN were significantly higher than those in patients with focal proliferative LN and membranous LN. The combination of plasma ADMA, complement C3, and eGFR may assist in the diagnosis of diffuse proliferative LN. Low levels of plasma ADMA are an independent protective factor for complete renal remission in patients with proliferative $\mathrm{LN}$ treated with cyclophosphamide as induction therapy.

\section{Acknowledgment}

The authors are deeply grateful to all of the patients and healthy volunteers who donated blood.

\section{Statement of Ethics}

All of the participants signed a written informed consent form for participation in this study. Ethics approval was obtained from the Medial Ethics Committee of Guangdong Provincial People's Hospital (No. GDREC 2001625H).

\section{Conflict of Interest Statement}

All of the authors declare no competing interests.

\section{Funding Sources}

This study was supported by the National Natural Science Foundation of China (grants 81770667 and 81970625), the Fundamental Research Funds for the Central Universities (grants D2180530), and the Natural Science Foundation of Guangdong Province (grants 2019A1515010286).

\section{Author Contributions}

X. Liang and X. Yu designed this study. L. Zhang and K. Zhang performed all of the experiments and wrote this paper. W. Dong revised this paper. R. Huang, H. Zhang, W. Shi, S. Liu, Z. Li, Y. Chen, and Z. Ye helped with clinical data collection. All of the authors read and approved the final version of this work. 


\section{References}

1 Yap DY, Tang CS, Ma MK, Lam MF, Chan TM. Survival analysis and causes of mortality in patients with lupus nephritis. Nephrol Dial Transplant. 2012 Aug;27(8):3248-54.

2 Lerang K, Gilboe IM, Steinar Thelle D, Gran JT. Mortality and years of potential life loss in systemic lupus erythematosus: a populationbased cohort study. Lupus. 2014 Dec;23(14): 1546-52.

3 Bernatsky S, Boivin JF, Joseph L, Manzi S, Ginzler E, Gladman DD, et al. Mortality in systemic lupus erythematosus. Arthritis Rheum. 2006 Aug;54(8):2550-7.

4 Faurschou M, Dreyer L, Kamper AL, Starklint $\mathrm{H}$, Jacobsen S. Long-term mortality and renal outcome in a cohort of 100 patients with lupus nephritis. Arthritis Care Res (Hoboken). 2010 Jun;62(6):873-80.

5 Alarcón GS. Multiethnic lupus cohorts: what have they taught us? Reumatol Clin. 2011 JanFeb;7(1):3-6.

6 Almaani S, Meara A, Rovin BH. Update on Lupus Nephritis. Clin J Am Soc Nephrol. 2017 May;12(5):825-35.

7 Tunnicliffe DJ, Palmer SC. Immunosuppressive Treatment for Proliferative Lupus $\mathrm{Ne}$ phritis: Summary of a Cochrane Review. Am J Kidney Dis. 2018 Nov;72(5):756-7.

8 Bertsias GK, Tektonidou M, Amoura Z, Aringer M, Bajema I, Berden JH, et al.; European League Against Rheumatism and European Renal Association-European Dialysis and Transplant Association. Joint European League Against Rheumatism and European Renal Association-European Dialysis and Transplant Association (EULAR/ERA-EDTA) recommendations for the management of adult and paediatric lupus nephritis. Ann Rheum Dis. 2012 Nov;71(11):1771-82.

9 Rovin BH, Caster DJ, Cattran DC, Gibson KL, Hogan JJ, Moeller MJ, et al.; Conference Participants. Management and treatment of glomerular diseases (part 2): conclusions from a Kidney Disease: Improving Global Outcomes (KDIGO) Controversies Conference. Kidney Int. 2019 Feb;95(2):281-95.

10 Soliman S, Mohan C. Lupus nephritis biomarkers. Clin Immunol. 2017 Dec;185:10-20.

11 Tain YL, Hsu CN. Toxic Dimethylarginines: Asymmetric Dimethylarginine (ADMA) and Symmetric Dimethylarginine (SDMA). Toxins (Basel). 2017 Mar;9(3):E92.

12 Oliva-Damaso E, Oliva-Damaso N, Rodriguez-Esparragon F, Payan J, BaamondeLaborda E, Gonzalez-Cabrera F, et al. Asymmetric (ADMA) and Symmetric (SDMA) Dimethylarginines in Chronic Kidney Disease:
A Clinical Approach. Int J Mol Sci. 2019 Jul; 20(15):E3668

13 Bultink IE, Teerlink T, Heijst JA, Dijkmans BA, Voskuyl AE. Raised plasma levels of asymmetric dimethylarginine are associated with cardiovascular events, disease activity, and organ damage in patients with systemic lupus erythematosus. Ann Rheum Dis. 2005 Sep;64(9):1362-5.

14 Kiani AN, Mahoney JA, Petri M. Asymmetric dimethylarginine is a marker of poor prognosis and coronary calcium in systemic lupus erythematosus. J Rheumatol. 2007 Jul;34(7): 1502-5.

15 Zoccali C, Bode-Böger S, Mallamaci F, Benedetto F, Tripepi G, Malatino L, et al. Plasma concentration of asymmetrical dimethylarginine and mortality in patients with end-stage renal disease: a prospective study. Lancet. 2001 Dec;358(9299):2113-7.

16 Fujimi-Hayashida A, Ueda S, Yamagishi S, Kaida Y, Ando R, Nakayama Y, et al. Association of asymmetric dimethylarginine with severity of kidney injury and decline in kidney function in IgA nephropathy. Am J Nephrol. 2011;33(1): 1-6.

17 Levey AS, Stevens LA, Schmid CH, Zhang YL, Castro AF 3rd, Feldman HI, et al.; CKD-EPI (Chronic Kidney Disease Epidemiology Collaboration). A new equation to estimate glomerular filtration rate. Ann Intern Med. 2009 May;150(9):604-12.

18 Weening JJ, D’Agati VD, Schwartz MM, Seshan SV, Alpers CE, Appel GB, et al. The classification of glomerulonephritis in systemic lupus erythematosus revisited. J Am Soc Nephrol. 2004 Feb;15(2):241-50.

19 Vallance P, Leone A, Calver A, Collier J, Moncada S. Endogenous dimethylarginine as an inhibitor of nitric oxide synthesis. J Cardiovasc Pharmacol. 1992;20 Suppl 12:S60-2.

20 Siroen MP, Teerlink T, Nijveldt RJ, Prins HA, Richir MC, van Leeuwen PA. The clinical significance of asymmetric dimethylarginine. Annu Rev Nutr. 2006;26(1):203-28.

21 Alpoim PN, Sousa LP, Mota AP, Rios DR, Dusse LM. Asymmetric dimethylarginine (ADMA) in cardiovascular and renal disease. Clin Chim Acta. 2015 Feb;440:36-9.

22 Liu X, Xu X, Shang R, Chen Y. Asymmetric dimethylarginine (ADMA) as an important risk factor for the increased cardiovascular diseases and heart failure in chronic kidney disease. Nitric Oxide. 2018 Aug;78:113-20.

23 Kajimoto H, Kai H, Aoki H, Yasuoka S, Anegawa T, Aoki Y, et al. Inhibition of eNOS phosphorylation mediates endothelial dys- function in renal failure: new effect of asymmetric dimethylarginine. Kidney Int. 2012 Apr;81(8):762-8.

24 Eiselt J, Rajdl D, Racek J, Vostrý M, Rulcová $\mathrm{K}$, Wirth J. Asymmetric dimethylarginine and progression of chronic kidney disease: a oneyear follow-up study. Kidney Blood Press Res. 2014;39(1):50-7.

25 Perna M, Roman MJ, Alpert DR, Crow MK, Lockshin MD, Sammaritano L, et al. Relationship of asymmetric dimethylarginine and homocysteine to vascular aging in systemic lupus erythematosus patients. Arthritis Rheum. 2010 Jun;62(6):1718-22.

$26 \mathrm{McDermott} J \mathrm{R}$. Studies on the catabolism of Ng-methylarginine, $\mathrm{Ng}$, Ng-dimethylarginine and $\mathrm{Ng}$, $\mathrm{Ng}$-dimethylarginine in the rabbit. Biochem J. 1976 Jan;154(1):179-84.

27 Torondel B, Nandi M, Kelly P, Wojciak-Stothard B, Fleming I, Leiper J. Adenoviral-mediated overexpression of DDAH improves vascular tone regulation. Vasc Med. 2010 Jun; 15(3):205-13.

28 Nijveldt RJ, Teerlink T, Siroen MP, van Lambalgen AA, Rauwerda JA, van Leeuwen PA. The liver is an important organ in the metabolism of asymmetrical dimethylarginine (ADMA). Clin Nutr. 2003 Feb;22(1): 17-22.

29 Nijveldt RJ, Teerlink T, van Guldener C, Prins $\mathrm{HA}$, van Lambalgen AA, Stehouwer CD, et al. Handling of asymmetrical dimethylarginine and symmetrical dimethylarginine by the rat kidney under basal conditions and during endotoxaemia. Nephrol Dial Transplant. 2003 Dec;18(12):2542-50.

30 Aldámiz-Echevarría L, Andrade F. Asymmetric dimethylarginine, endothelial dysfunction and renal disease. Int J Mol Sci. 2012;13(9): 11288-311.

31 Matsumoto Y, Ueda S, Yamagishi S, Matsuguma K, Shibata R, Fukami K, et al. Dimethylarginine dimethylaminohydrolase prevents progression of renal dysfunction by inhibiting loss of peritubular capillaries and tubulointerstitial fibrosis in a rat model of chronic kidney disease. J Am Soc Nephrol. 2007 May;18(5): 1525-33.

32 Yu F, Haas M, Glassock R, Zhao MH. Redefining lupus nephritis: clinical implications of pathophysiologic subtypes. Nat Rev Nephrol. 2017 Aug; 13(8):483-95.

33 Tojo A, Welch WJ, Bremer V, Kimoto M, Kimura K, Omata M, et al. Colocalization of demethylating enzymes and NOS and functional effects of methylarginines in rat kidney. Kidney Int. 1997 Dec;52(6):1593-601. 